\title{
Disease Characteristics and the Burden of Joint and Skin Involvement Amongst People With Psoriatic Arthritis: A Population Survey
}

\author{
William Tillett · Joseph F. Merola • Diamant Thaçi • Elizabeth Holdsworth • \\ Nicola Booth (D) - L. Steve Lobosco - Gary Milligan - Matthew M. Hufford • \\ Julie A. Birt · Wolf-Henning Boehncke
}

Received: May 6, 2020 / Published online: July 22, 2020

(C) The Author(s) 2020

\section{ABSTRACT}

Introduction: Psoriatic arthritis (PsA) is a chronic, systemic, inflammatory disease where disease burden and quality of life (QoL) are affected by both joint and skin manifestations. Methods: Patient and physician reported data were collected about 3200 patients in a crosssectional survey of patients from nine countries. Patient-reported outcomes (PROs) included perceptions of symptom importance, EuroQol questionnaire (EQ-5D), Psoriatic Arthritis

Electronic supplementary material The online version of this article (https://doi.org/10.1007/s40744020-00221-8) contains supplementary material, which is available to authorized users.

Digital Features To view digital features for this article go to: https://doi.org/10.6084/m9.figshare.12546644.

W. Tillett

Royal National Hospital for Rheumatic Diseases, Bath, UK

\section{W. Tillett}

Pharmacy and Pharmacology, University of Bath, Bath, UK

J. F. Merola

Brigham and Women's Hospital, Harvard Medical

School, Boston, MA, USA

D. Thaçi

Institute and Comprehensive Center Inflammation

Medicine, University of Lübeck, Lübeck, Germany
Impact of Disease (PsAID12), and Work Productivity and Activity Impairment (WPAI) Index. Outcomes were compared in patients with 'joint-only' and 'joint and skin' disease symptoms.

Results: Of the 3200 patients, 2703 had complete information for 'joint-only' or 'joint and skin' involvement and were included in the analysis. Patients had a mean age of 49.2 years, $45.2 \%$ were female, and $64.5 \%$ had 'joint and skin' involvement. Patients with 'joint and skin' involvement had higher mean tender and swollen joint counts (5.2 and 4.8) than patients who were 'joint-only' (2.0 and 1.5). Significantly more patients with active 'joint and skin' symptoms experienced a flare (currently or within the last 12 months) compared with 'joint-only' patients (34.9 vs. 23.2\%, $p<0.001$ ). When asked to prioritize the burden of

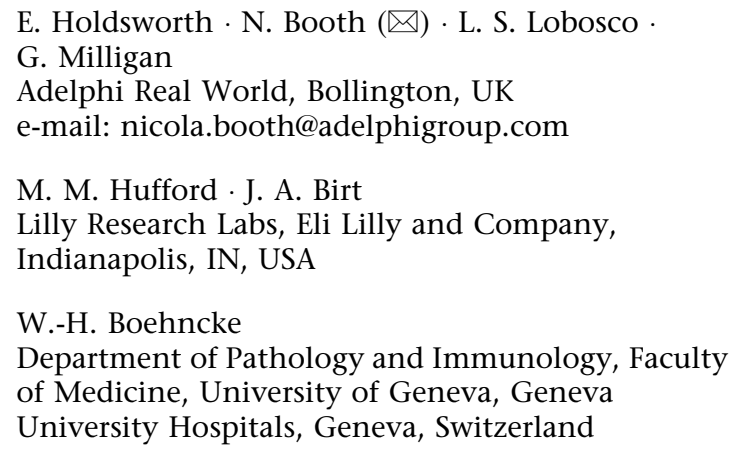


symptoms, $61.6 \%$ of patients prioritized joints, $38.4 \%$ prioritized skin. Anxiety/depression was experienced by $41.4 \%$ of patients, $62.4 \%$ of whom indicated that both joint and skin symptoms were the cause. Patients with 'joint and skin' involvement reported significantly worse QoL, work productivity and activity impairment than 'joint-only' patients (EQ-5D index 0.79 vs. $0.85, p<0.001$; EQ-5D VAS 71.98 vs. $77.68, p<0.001$; PsAID12 2.91 vs. 1.66 , $p<0.001$; WPAI overall work impairment 25.61 vs. $16.32, p<0.001)$.

Conclusions: PsA patients who experience 'joint and skin' symptoms had significantly worse clinical outcomes, health-related QoL, and work productivity compared with patients with 'joint-only' symptoms.

Keywords: HR-QoL; Psoriatic arthritis; Realworld evidence; Work productivity

\section{Key Summary Points}

Psoriatic arthritis (PsA) patients are impacted by joint involvement and skin involvement to varying degrees.

A comparison of 2703 patients with 'jointonly' and 'joint and skin' manifestations of PsA, in a real-world cross-sectional survey in nine countries.

Patients with 'joint and skin' symptoms experience worse clinical outcomes than joint-only patients - higher joint counts, more symptoms, more patients experience flares.

Patients with 'joint and skin' involvement reported significantly worse quality of life, work productivity, and activity impairment (EQ-5D, PsAID12 and WPAI, all $p<0.001$ ) than those with joint-only PsA.

Future treatment options should focus on both joint and skin symptoms in order to optimize patient outcomes.

\section{INTRODUCTION}

Psoriatic arthritis (PsA) is a systemic inflammatory disease that occurs in up to $30 \%$ of patients with psoriasis, and is characterized by joints that are swollen, painful, and stiff [1-3]. PsA is differentiated from other forms of inflammatory arthritis by the presence of other clinical features including enthesitis, dactylitis, axial disease, and psoriatic skin and nail lesions. PsA is a chronic and progressive condition that can result in joint damage and deformities if not treated early $[4,5]$. The relationship between the joint and skin components of PsA and their relative contribution to patient burden of disease has not been extensively researched, although several studies have shown that both articular and dermatologic manifestations contribute to overall patient burden in PsA patients $[6,7]$.

Patients with PsA have significantly poorer health-related quality of life (QoL) than the general population in multiple studies conducted worldwide [8-10]. Patients with PsA have been shown to have similar levels of impaired QoL as patients with rheumatoid arthritis despite less overall peripheral joint disease severity in PsA $[9,11]$. This additional QoL impact is often attributed to the presence of concurrent skin manifestations. Observational studies of patients with PsA have shown that greater skin severity is accompanied by increased disease activity including a greater degree of joint involvement. Furthermore, greater skin severity is associated with worse patient reported outcomes (PROs) including lower physical functioning, more patient-reported pain and fatigue, and a worse quality of life $[6,7,12,13]$.

Patients with PsA can experience a significant mental health impact related to their disease, including anxiety and depression. Limited data are available but one recent systematic literature review found PsA patients can develop anxiety and depression at a rate higher than the general population [14]. The prevalence of depression ranged from 9 to $22 \%$ and anxiety from 15 to $30 \%$ in PsA patients but whether joint symptoms or skin symptoms have greater 
contribution, or if they contribute equally to these effects has not been well studied in a realworld setting. Patients with PsA can also experience acute exacerbations of disease, either as a joint or skin flare, or have variable involvement of both components whose pattern and impact on patients have not been fully quantified. A formal definition of a flare in PsA has yet to be developed $[15,16]$.

The objective of this cross-sectional study from a real-world population of patients with PsA was to assess and quantify the burden of joint and skin symptoms to PsA patients, including their role in flares, contribution to emotional burden, and impact on PROs through an observational study of patients from nine countries across the Asia-Pacific, Europe, and the United States.

\section{METHODS}

A large-scale cross-sectional survey using established methods [17] was conducted with rheumatologists and dermatologists and their patients with PsA across Australia, Canada, France, Germany, Italy, Japan, Spain, UK, and the US. In Japan, the specialty of physicians participating in the study also included orthopedic surgeons and internal medicine. The data were collected from April to November 2018.

\section{Study Design and Population}

The study was a non-interventional, cross-sectional survey that captured current and historic data from physicians on their consulting PsA patients and patient-reported outcomes. Physicians were identified via publicly available lists and invited to participate in the study. Those who agreed to participate were asked to complete a patient record form for a minimum of the next three adult patients they consulted with who had a physician-confirmed diagnosis of PsA, were aged $\geq 18$ years, and for whom they were actively involved in drug management. The same patients were invited, by their physicians, to complete a voluntary questionnaire. Clinical diagnosis of PsA was based upon the physicians' individual judgment with no restrictions on how the diagnosis was determined for inclusion in the study.

\section{Data Collection}

This study used data derived from physicianreported patient record forms (PRFs) and voluntary patient self-completed forms (PSCs). Physician-reported data included patient demographics, clinical characteristics including PsA symptoms, flaring patterns, and clinical outcome measures. Physicians also provided information on the number of joints involved in the patient's PsA, and assessed overall, joint, and skin symptom severity by rating as either 'None', 'Mild', 'Moderate', or 'Severe', based on their own clinical judgement. Patient-reported data included information on general health, disease history and symptoms, medications, and patient-reported outcome measures (EuroQoL 5D) [18, 19], Work Productivity and Activity Impairment (WPAI) [20, 21], Health Assessment Questionnaire-Disability Index (HAQ-DI) [22, 23], and the Psoriatic Arthritis Impact of Disease (PsAID12) questionnaire [24]). The EQ-5D utility score is derived from answers to five different areas that include mobility, self-care, usual activities, pain/discomfort, and anxiety/depression and is converted to a score that is anchored at 0 for death and 1 for perfect health. The VAS allows respondents to report their perceived health status with a grade ranging from 0 (the worst possible health status) to 100 (the best possible health status). The HAQ-DI is an index measuring the quality of life related to health originally designed for assessment of patients with rheumatoid arthritis and has values from $0-3$ where a value of 0 to 1 indicates mild difficulties to moderate disability, from 1 to 2 : moderate to severe disability and from 2 to 3 severe to very severe disability. The PsAID is a PsA-specific instrument developed by the European League Against Rheumatism (EULAR) that provides a patient-reported measure of disease impact on life in general. The questionnaire uses a weighted scoring system, has a range of $0-10$ with 4 being considered a patient acceptable symptom state $[24,25]$. 
In order to assess the impact of joint and skin symptoms, PsA patients were also asked a short series of questions; 'Given the choice of only one, would you rather be completely free of your...? [Skin symptoms; Joint symptoms]', 'Do you experience anxiety and/or depression as a direct result of your PsA?', 'Which of the following is the cause of your anxiety and/or depression? [Your skin symptoms; Your joint symptoms; Both your skin and joint symptoms]'.

\section{Endpoints, Data Analysis, and Statistical Methods}

To be eligible for inclusion in this analysis, physician-reported patient data had to include confirmation of the presence of skin psoriasis currently or historically, and/or have current BSA information available. Patients were grouped during the analysis according to whether they experienced joint symptoms alone, or joint symptoms plus active skin symptoms. Patients were defined as having 'joint-only' PsA if the treating physician reported body surface area affected by psoriasis was currently $0 \%$ or provided a response of 'no diagnosis of psoriasis'. Similarly, they were defined as having 'joint and skin' PsA if their current BSA was $>0 \%$. A sensitivity analysis was also conducted by grouping patients according to the number of affected joints ( $\leq 4$ joints vs. $>4$ joints) and for severity of skin symptoms (mild, moderate, severe). The number of affected joints was derived from asking the physician 'How many joints are currently affected by this patient's PsA?' while the severity of skin symptoms stemmed from physician subjective assessment.

Physicians provided further estimates of the impact of PsA using a VAS and where this data was available the score on a scale of $0-100$ was based on 0 being equivalent to the best possible health assessment and 100 the worst health assessment. This was collected for global, skin, and joint symptoms separately. Pain was assessed by a physician-reported numeric rating scale (NRS, range 1-10) with 1 representing no pain and 10 worst possible pain. Flaring was assessed by physicians reporting if the patient ever experiences flares (acute episodes), and their response based on their own subjective assessment-no formal definitions of flaring were provided.

Statistical analysis was performed using Stata (version 15.1). Patient demographics, clinical characteristics and PROs were described within each subgroup (joints \& skin vs. joints only and $\leq 4$ joints vs. $>4$ joints for the sensitivity analysis) and overall, using $n$, mean and standard deviation (for numeric variables) or $n$ and percent (for categorical variables). Clinical burden and PROs were also described ( $n$, mean, SD, \%) and compared between physician-reported skin severity (mild, moderate or severe).

When two groups were compared, numeric variables were compared using a $t$ test; ordered categorical variables were compared using a Mann-Whitney test; binary categorical variables were compared using a Fisher's exact test; other categorical variables were compared using a Chi-squared test. When more than two groups were compared, numeric variables were compared using an ANOVA; ordered categorical variables were compared using a Kruskal-Wallis test; categorical variables were compared using a Chi-squared test. $p$ values $<0.05$ were considered statistically significant.

\section{Ethical Considerations}

The non-interventional, observational nature of the data collection does not result in patients being placed at risk from the study. Patients provided informed consent to participate in the study and did not provide any personally identifiable information. All responses were anonymized to preserve respondent (physician and patient) confidentiality and all participating physicians and patients were assigned a study number to aid anonymous data collection and to allow linkage of data during data collection and analysis.

The research was conducted in accordance with national market research and privacy regulations (EphMRA, U.S. Department of Health and Human Services National Institutes of Health, HIPAA). Ethical approvals were sought 
and granted through Western-IRB (Study ID number 1183030) for the US \& Canada, and through Freiburg Ethics Commission (FEKI) for all other countries.

\section{RESULTS}

Data were collected for patients with PsA by 454 rheumatologists and 238 dermatologists for 3200 patients (1514 rheumatologist patients; 1686 dermatologist patients) from nine countries representing Asia Pacific (APAC, $n=443$; 13.8\%), Europe (EU5, $n=1966 ; 61.4 \%$ ), and North America $(n=791 ; 24.7 \%)$. Body surface area information was available for 2540 patients of whom $797(31.4 \%)$ had a BSA score of 0 while $1743(68.6 \%)$ had a score of greater than 0 . These patients $(n=2540)$, and patients with no diagnosis of psoriasis $(n=163,5.1 \%)$ are the focus of this analysis and gave a total population size of 2703 (Table 1).

\section{Patient Demographic and Clinical Characteristics}

Across the 2703 PsA patients, $54.8 \%$ were male, with a mean (SD) age of 49.2 (13.4) years, and a mean (SD) BMI of 26.9 (6.7). 'Joint only' and 'joint and skin' patients were comparable demographically, except in the distribution of employment status (Table 1).

In the overall population, most patients had mild severity disease reported by physicians (Table 2) with a mean of 1.83 current symptoms, 3.19 affected joints, and a pain score of 2.75. Physician-determined overall disease severity was worse in patients with 'joint and skin' involvement than those with 'joint only' PsA, with a significantly higher proportion reported as moderate or severe (30.7 vs. $14.2 \%$, $p<0.001$ ) (Table 2). Additionally, the mean number of affected joints was 4.0 in patients with 'joint and skin' involvement and was significantly higher than in patients with joint only PsA where a value of 1.8 was seen $(p<0.001)$. This picture of detrimental clinical characteristics in patients with 'joint and skin' symptoms extended to physician assessed disease activity including global, joint, and skin VAS scores (Table 2).

Both groups of patients received csDMARDs in approximately comparable percentages (34.3 and $30.6 \%$, respectively), however, there were significant differences in the percentage of patients receiving targeted synthetic DMARDs or biologic DMARDs in the two categories. Patients with 'joint only' PsA were significantly less likely to be currently receiving tsDMARDs (apremilast or tofacitinib) compared with patients with 'joint and skin' symptoms (6.9 vs. $11.5 \%, p=0.001$ ). Conversely, patients with joint only symptoms were more likely to receive biologic DMARDs compared with patients with skin and joint symptoms (67.4 vs. $54.0 \%$, $p<0.001$ ) (Table 1).

\section{Flaring Patterns}

Physician-reported flaring was available for 2665 patients and suggested that $58.9 \%$ of PsA patients experienced flares at any time (Table 3) with a greater percentage of patients with active 'joint and skin' symptoms experiencing a flare currently, within the last 12 months or at periods longer than 12 months compared with 'joint-only' patients (62.3 vs. $52.7 \%)$. In the overall population, the mean number of flares was 0.65 in the last 12 months and did not vary significantly in patients with 'joint only' (0.60) and joint and skin symptoms (0.68). The mean duration of a flare was 17.4 days in the overall population and again did not vary significantly with symptoms being 17.8 days in joint only patients and 17.3 days in joint and skin patients.

Physicians reported that in the overall population 197 patients (7.4\%) currently had a flare with the current flare severity assessed as mild $(18.3 \%)$, moderate $(62.4 \%)$, and severe (19.3\%). When assessed in terms of 'joint only' and 'joint and skin' status there were no significant differences between groups with the current flare assessed as mild in $27.7 \%$ of joint only patients versus $15.3 \%$ of joint and skin patients. Corresponding values for moderate and severe flares were 57.4 vs. $64.0 \%$ and 14.9 vs. $20.7 \%$. 
Table 1 Baseline demographics in patients according to 'joint-only' and 'joint and skin' PsA

\begin{tabular}{|c|c|c|c|c|}
\hline Parameter & Overall & Joint-only & Joint and skin & $p$ value \\
\hline$n, \%$ & $2703(100.0)$ & $960(35.5)$ & $1743(64.5)$ & \\
\hline Managed by rheumatologist, $n(\%)$ & $1082(40.0)$ & $440(45.8)$ & $642(36.8)$ & \\
\hline \multicolumn{5}{|l|}{ Age } \\
\hline Mean (SD) & $49.2(13.4)$ & $49.3(13.6)$ & $49.1(13.3)$ & \multirow[t]{2}{*}{$0.697^{*}$} \\
\hline Median (IQR) & $49.0(39.0,58.0)$ & $49.0(40.0,59.0)$ & $49.0(39.0,58.0)$ & \\
\hline Male, $n(\%)$ & $1480(54.8)$ & $531(55.3)$ & $949(54.4)$ & $0.686^{\#}$ \\
\hline \multicolumn{5}{|l|}{ BMI } \\
\hline Mean (SD) & $26.9(6.7)$ & $27.0(6.1)$ & $26.8(7.0)$ & \multirow[t]{2}{*}{$0.321^{*}$} \\
\hline Median (IQR) & $25.9(23.4,28.7)$ & $25.9(23.4,29.4)$ & $25.8(23.3,28.4)$ & \\
\hline \multicolumn{5}{|l|}{ Employment status, $n(\%)$} \\
\hline$N$ & 2605 & 914 & 1691 & \multirow[t]{6}{*}{$<0.001^{\ddagger}$} \\
\hline Working full time & $1497(57.5)$ & $554(60.6)$ & $943(55.8)$ & \\
\hline Working part time & $283(10.9)$ & $82(9.0)$ & $201(11.9)$ & \\
\hline Retired & $361(13.9)$ & $142(15.5)$ & $219(13.0)$ & \\
\hline Unemployed & $133(5.1)$ & $35(3.8)$ & $98(5.8)$ & \\
\hline Other & $331(12.7)$ & $101(11.1)$ & $230(13.6)$ & \\
\hline \multicolumn{5}{|l|}{ Charlson Comorbidity Index } \\
\hline Mean (SD) & $0.24(1.73)$ & $0.22(1.70)$ & $0.25(1.75)$ & \multirow[t]{2}{*}{$0.612^{*}$} \\
\hline Median (IQR) & $0.00(0.0,0.0)$ & $0.00(0.0,0.0)$ & $0.00(0.0,0.0)$ & \\
\hline \multicolumn{5}{|c|}{ Treatment classes currently receiving, $n$ (\%) } \\
\hline csDMARD & $863(31.9)$ & $329(34.3)$ & $534(30.6)$ & $0.058^{\#}$ \\
\hline tsDMARD & $267(9.9)$ & $66(6.9)$ & $201(11.5)$ & $<0.001^{\#}$ \\
\hline bDMARD & $1589(58.8)$ & $647(67.4)$ & $942(54.0)$ & $<0.001^{\#}$ \\
\hline
\end{tabular}

For employment status 'other' represents sum of homemaker, student and on long-term sick leave $B M I$ body mass index; IQR interquartile range; $S D$ standard deviation; csDMARD conventional synthetic DMARD; tsDMARD, targeted synthetic DMARD (apremilast or tofacitinib); $b D M A R D$, biologic DMARD

${ }^{*}$ Student's $t$ test; ${ }^{\#}$ Fisher's exact test; ${ }^{\ddagger}$ Chi-squared test were used when comparing outcomes from 'joint-only' with 'joint and skin' groups

Of patients currently flaring, $47.7 \%$ of patients indicated that both their skin and joints were currently worse than normal; $40 \%$ reported only their joints were worse than normal while only $8.4 \%$ reported only their skin was worse than normal.

\section{Patient Perception of Relative Importance of Their Joint and Skin or Joint Only Symptoms}

A total of 573 patients provided an assessment of mental health burden associated with PsA. 
Table 2 Clinical characteristics of patients in patients stratified according to 'joint-only' and 'joint and skin' PsA

\begin{tabular}{|c|c|c|c|c|}
\hline Parameter & Overall & Joint-only & Joint and skin & $p$ value \\
\hline$n(\%)$ & $2703(100.0)$ & $960(35.5)$ & $1743(64.5)$ & \\
\hline Diagnosed with psoriasis, $n(\%)$ & 2540 & 797 & 1743 & \\
\hline $\mathrm{BSA}=0$ & $797(31.4)$ & $797(100.0)$ & $0(0.0)$ & \\
\hline $\mathrm{BSA}>0$ to $\leq 3$ & $670(26.3)$ & $0(0.0)$ & $670(38.4)$ & \\
\hline $\mathrm{BSA}>3$ to $<10$ & $515(20.2)$ & $0(0.0)$ & $515(29.5)$ & \\
\hline $\mathrm{BSA} \geq 10$ & $561(22.1)$ & $0(0.0)$ & $561(32.1)$ & \\
\hline BSA, mean $(S D)$ & - & - & $9.4(10.88)$ & \\
\hline \multicolumn{5}{|l|}{ Time since PsA diagnosis, months } \\
\hline Mean (SD) & $58.16(71.54)$ & $69.27(77.64)$ & $52.50(67.54)$ & $<0.001^{\#}$ \\
\hline Median (IQR) & $33.75(13.73,73.66)$ & $39.98(16.93,93.96)$ & $29.00(12.35,63.99)$ & \\
\hline \multicolumn{5}{|l|}{ Overall severity, $n$ (\%) } \\
\hline Mild & $2031(75.1)$ & $824(85.8)$ & $1207(69.2)$ & $<0.001^{*}$ \\
\hline Moderate & $597(22.1)$ & $124(12.9)$ & $473(27.1)$ & \\
\hline Severe & $75(2.8)$ & $12(1.3)$ & $63(3.6)$ & \\
\hline \multicolumn{5}{|l|}{ Joint severity, $n$ (\%) } \\
\hline Mild & $2044(75.6)$ & $822(85.6)$ & $1222(70.1)$ & $<0.001^{*}$ \\
\hline Moderate & $588(21.8)$ & $125(13.0)$ & $463(26.6)$ & \\
\hline Severe & $71(2.6)$ & $13(1.4)$ & $58(3.3)$ & \\
\hline \multicolumn{5}{|l|}{ Number of current symptoms } \\
\hline Mean (SD) & $1.83(1.72)$ & $1.03(1.35)$ & $2.27(1.74)$ & $<0.001^{\#}$ \\
\hline Median (IQR) & $2.00(0.00,3.00)$ & $1.00(0.00,2.00)$ & $2.00(1.00,3.00)$ & \\
\hline \multicolumn{5}{|l|}{ Number of joints affected } \\
\hline Mean (SD) & $3.19(4.57)$ & $1.78(3.85)$ & $3.97(4.74)$ & $<0.001^{\#}$ \\
\hline Median (IQR) & $2.00(0.00,4.00)$ & $0.00(0.00,2.00)$ & $3.00(1.00,5.00)$ & \\
\hline \multicolumn{5}{|l|}{66 Swollen joint count } \\
\hline$n$ & 587 & 238 & 349 & $<0.001^{*}$ \\
\hline Mean (SD) & $3.48(7.47)$ & $1.50(3.45)$ & $4.83(9.03)$ & \\
\hline Median (IQR) & $1.00(0.00,4.00)$ & $0.00(0.00,2.00)$ & $2.00(0.00,6.00)$ & \\
\hline \multicolumn{5}{|l|}{68 Tender joint count } \\
\hline$n$ & 557 & 221 & 336 & $<0.001^{*}$ \\
\hline Mean (SD) & $3.92(6.64)$ & $1.97(3.67)$ & $5.20(7.76)$ & \\
\hline Median (IQR) & $2.00(0.00,4.00)$ & $1.00(0.00,2.00)$ & $3.00(1.00,6.00)$ & \\
\hline
\end{tabular}


Table 2 continued

\begin{tabular}{lllll}
\hline Parameter & \multicolumn{1}{c}{ Overall } & Joint-only & Joint and skin & p value \\
\hline $\begin{array}{l}\text { Physician assessed Global VAS score } \\
n\end{array}$ & 405 & 145 & 260 & \\
Mean (SD) & $23.36(22.74)$ & $17.93(21.51)$ & $26.39(22.88)$ & $<0.001^{\#}$ \\
Physician-assessed skin VAS score & & & \\
$n$ & 136 & 35 & 101 & $<0.001^{\#}$ \\
Mean (SD) & $14.21(16.28)$ & $3.14(7.03)$ & $18.05(16.83)$ & \\
Physician-assessed joint VAS score & & & $0.002^{\#}$ \\
$n$ & 204 & 67 & 137 & \\
Mean (SD) & $21.11(22.59)$ & $14.01(17.00)$ & $24.58(24.18)$ & \\
Physician-reported pain $(1-10)$ & & 1743 & \\
$n$ & 2703 & 960 & $3.08(1.79)$ & \\
Mean (SD) & $2.75(1.75)$ & $2.16(1.51)$ & $3.00(2.00,4.00)$ & \\
Median (IQR) & $2.00(1.00,4.00)$ & $2.00(1.00,3.00)$ & & \\
\hline
\end{tabular}

$I Q R$ interquartile range; $S D$ standard deviation

*Mann-Whitney test; "Student's $t$ test were used when comparing outcomes from 'joint-only' with 'joint and skin' groups

Over $41 \%$ of patients indicated that they currently experienced anxiety/depression as a result of their PsA (Fig. 1).

There was a significant difference in the patient-reported cause of their anxiety/depression with 'both joint and skin symptoms' being mentioned by $56.1 \%$ of 'joint only' patients and $65.0 \%$ of 'joint and skin' patients. Skin symptoms were the cause of anxiety/depression in 3.0\% of joint only patients and $11.7 \%$ of joint and skin patients, while joint symptoms were the cause of anxiety/ depression in $40.9 \%$ of joint only patients and $23.3 \%$ of joint and skin patients.

Based on the question "Given the choice of only one, would you rather be completely free of your skin symptoms or joint symptoms?", $61.6 \%$ of patients prioritized joint symptoms, while $38.4 \%$ prioritized skin symptoms. The symptom prioritization did not differ significantly ( $p=0.1513$ ) between the 'joint only' and 'joint and skin' patient groups with 64.7 and $60.2 \%$, respectively, prioritizing joint symptoms. Even in patients with a BSA $=0$ or 'no diagnosis of psoriasis', $35.3 \%$ of patients in the 'joint only' group prioritized skin symptoms as most important compared with $39.8 \%$ of patients in the 'joint and skin' group (Fig. 1). Joint and skin patients managed by rheumatologists prioritize the symptoms in a similar way (Supplementary Table 1), and results are consistent when analyzed on a regional basis (Supplementary Fig. 1).

\section{Patient-Reported Outcomes}

Across a range of PROs, patients with 'joint and skin' involvement showed significantly worse outcomes compared with patients with 'jointonly' symptoms. Physical function measured by the HAQ-DI was statistically significantly worse in the 'joint and skin' group compared to the 'joint-only' group (0.57 vs. 0.33, $p<0.001)$. Likewise, quality of life and health status as assessed by the EQ5D and PsAID questionnaires were also statistically significantly worse in the 'joint and skin' group compared to the 'jointonly' group. The mean (SD) EQ-5D index was 0.85 (0.17) in the 'joint only' group and 0.79 (0.17) in patients with 'joint and skin' involvement (Table 4). A similar pattern was seen with 
Table 3 Experience of flares, their severity, and location in patients with PsA (physician-reported)

\begin{tabular}{|c|c|c|c|c|}
\hline Parameter & Overall & Joint-only & Joint and skin & $p$ value \\
\hline \multicolumn{5}{|l|}{ Does the patient experience flares? $n(\%)$} \\
\hline$n$ & 2665 & 955 & 1710 & $<0.001^{*}$ \\
\hline Yes, currently experiencing a flare & $197(7.4)$ & $47(4.9)$ & $150(8.8)$ & \\
\hline Yes, within the last 12 months but not currently & $621(23.3)$ & $175(18.3)$ & $446(26.1)$ & \\
\hline Yes, but not in the last 12 months & $751(28.2)$ & $282(29.5)$ & $469(27.4)$ & \\
\hline No & $1096(41.1)$ & $451(47.2)$ & $645(37.7)$ & \\
\hline \multicolumn{5}{|l|}{ Severity of current flare, $n(\%)$} \\
\hline$n$ & 197 & 47 & 150 & $0.147^{*}$ \\
\hline Mild & $36(18.3)$ & $13(27.7)$ & $23(15.3)$ & \\
\hline Moderate & $123(62.4)$ & $27(57.4)$ & $96(64.0)$ & \\
\hline Severe & $38(19.3)$ & $7(14.9)$ & $31(20.7)$ & \\
\hline \multicolumn{5}{|l|}{ Component currently flaring, $n(\%)$} \\
\hline$n$ & 197 & 47 & 150 & $<0.001^{*}$ \\
\hline Joints & $90(45.7)$ & $41(87.2)$ & $49(32.7)$ & \\
\hline Both joints and skin & $95(48.2)$ & $6(12.8)$ & $89(59.3)$ & \\
\hline Skin & $12(6.1)$ & $0(0.0)$ & $12(8.0)$ & \\
\hline \multicolumn{5}{|l|}{ Usual flaring component, $n$ (\%) } \\
\hline$n$ & 1569 & 504 & 1065 & $<0.001^{*}$ \\
\hline Joints & $582(37.1)$ & $268(53.2)$ & $314(29.5)$ & \\
\hline Both joints and skin & $795(50.7)$ & $176(34.9)$ & $619(58.1)$ & \\
\hline Skin & $192(12.2)$ & $60(11.9)$ & $132(12.4)$ & \\
\hline
\end{tabular}

Number of flares in the last 12 months

n

Mean (SD)

Median (IQR)

Typical length of flares (days)

n

Mean (SD)

Median (IQR)

2665955

$0.65(2.64)$

$0.00(0.00,1.00)$

$0.60(4.06)$

$0.00(0.00,0.00)$

1569

$17.4(16.4)$

$14.0(7.0,21.0)$
504

$17.8(18.4)$

$14.0(7.0,24.5)$
1710

$0.464^{\#}$

$0.68(1.30)$

$0.00(0.00,1.00)$

1065

$0.566^{\#}$

$17.3(15.4)$

$14.0(7.0,21.0)$

$I Q R$ interquartile range; $S D$ standard deviation

Chi-squared test; "Student's $t$ test were used when comparing outcomes from 'joint-only' with 'joint and skin' groups 


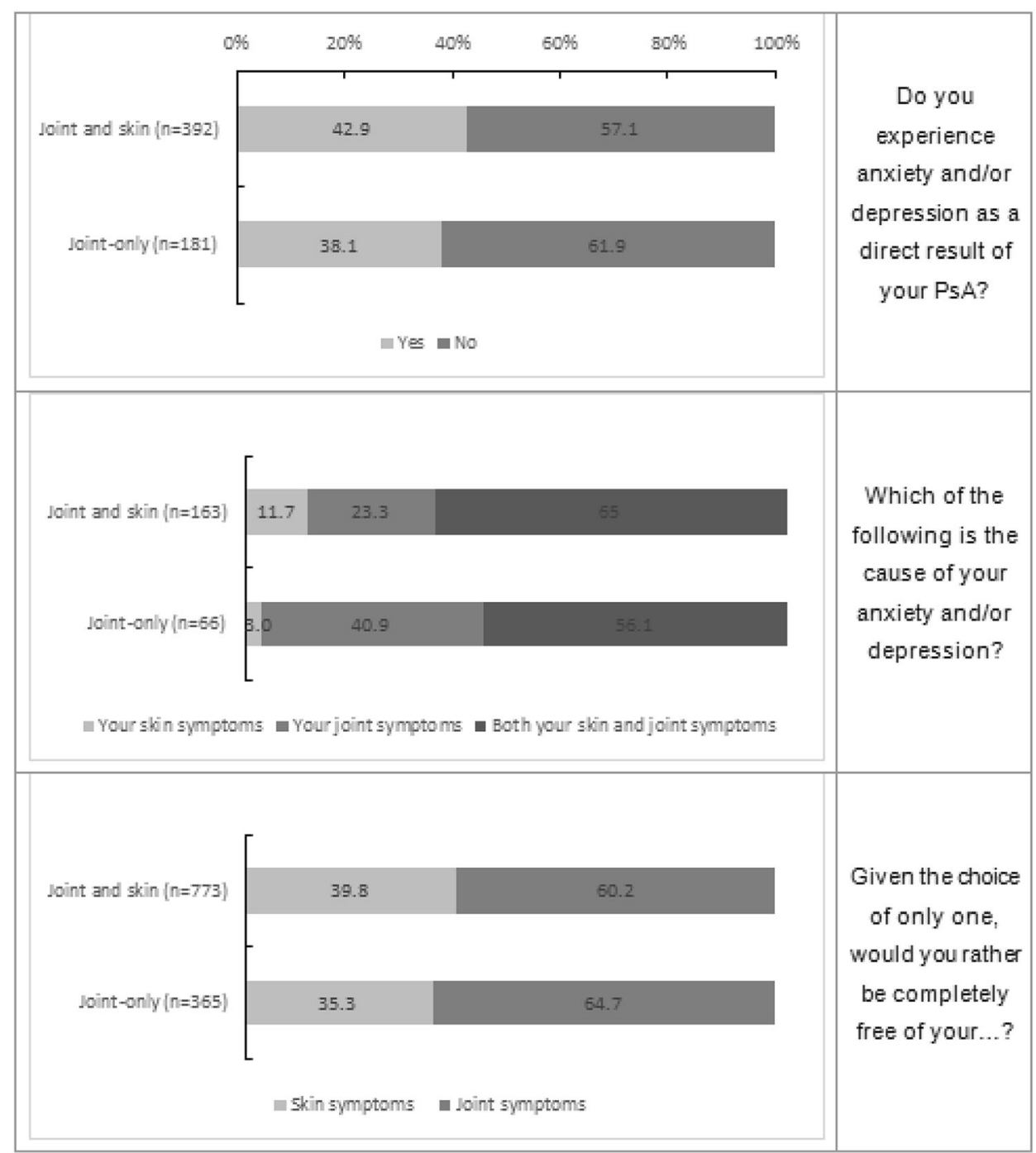

Fig. 1 Anxiety and depression, patient-reported causes of burden of symptoms in patients with 'joint-only' and 'joint and skin' symptoms. No significant differences $(p>0.001)$ were observed between patients with 'joint-only' and 'joint and skin' symptoms in response to the questions 'Do you

the EQ-5D visual analogue scale where the respective values were 77.68 (17.51) and 71.98 (19.01) and in both cases differences between the two groups were significant $(p<0.001)$.

The mean PsAID12 score (SD) was significantly higher at 2.91 (2.08) in patients with 'joint and skin' involvement compared with 1.66 (1.86) in patients with 'joint-only' involvement. For each of the 12 questions making up this validated score, patients with 'joint and skin' involvement showed experience anxiety and/or depression as a direct result of your PsA', 'What is the cause of your anxiety and/or depression' and 'Given the choice of only one, would you rather be free of your skin or joint symptoms?'

significantly higher mean scores compared with patients with 'joint-only' symptoms $(p<0.0001$ across all scores) (Fig. 2).

Significantly more work impairment was seen in three out of the four WPAI domains in patients with 'joint and skin' involvement compared with those with 'joint-only' involvement (Table 4).

PRO results are presented regionally in Supplementary Tables 4, 5, 6, and follow the same directional trend to the global results. 
Table 4 Patient-reported outcomes in overall population and patients with 'joint only' and 'joint and skin' PsA

\begin{tabular}{lllll}
\hline Parameter & Overall & Joint-only & Joint and skin & $p$ value \\
\hline EQ-5D utility score & & & & \\
$n$ & 1214 & 384 & 830 & $<.001$ \\
Mean (SD) & $0.81(0.18)$ & $0.85(0.17)$ & $0.79(0.17)$ & \\
Median (IQR) & $0.83(0.73,1.00)$ & $0.88(0.77,1.00)$ & $0.81(0.72,0.91)$ & \\
EQ-5D VAS & & & & \\
$n$ & 1228 & 389 & 839 & \\
Mean (SD) & $73.79(18.73)$ & $77.68(17.51)$ & $71.98(19.01)$ & \\
Median (IQR) & $80.00(65.0,90.0)$ & $80.00(70.0,90.0)$ & $75.00(60.0,85.0)$ & \\
HAQ Disability Index & & & 805 & \\
$n$ & 1174 & 369 & $0.57(0.59)$ &
\end{tabular}

WPAI: percent work time missed due to problem

$\begin{array}{llll}n & 567 & 186 & 381 \\ \text { Mean (SD) } & 5.11(16.68) & 5.86(17.64) & 4.74(16.20) \\ \text { Median (IQR) } & 0.00(0.00-0.00) & 0.00(0.00-0.00) & 0.00(0.00-0.00)\end{array}$

WPAI: percent impairment while working due to problem

$\begin{array}{lllll}n & 630 & 219 & 411 & <0.001 \\ \text { Mean (SD) } & 19.29(18.65) & 11.83(15.75) & 23.26(18.87) & \\ \text { Median (IQR) } & 20.00(0.00,30.00) & 10.00(0.00,20.00) & 20.00(10.00,30.00)\end{array}$

WPAI: percent overall work impairment due to problem

$\begin{array}{lllll}n & 556 & 183 & 373 & <0.001 \\ \text { Mean (SD) } & 22.56(20.99) & 16.32(20.64) & 25.61(20.51) & \\ \text { Median (IQR) } & 20.00(0.00,30.00) & 10.00(0.00,30.00) & 20.00(10.00,30.00) & \\ \text { WPAI: percent activity } & \text { impairment due to problem } & & & \\ n & 1204 & 385 & 819 & \\ \text { Mean (SD) } & 26.63(23.18) & 19.48(22.33) & 29.99(22.81) & <001 \\ \text { Median (IQR) } & 20.00(10.00,40.00) & 10.00(0.00,30.00) & 30.00(10.00,40.00) & \\ \text { PsAID12 score (0-10) } & & & 667 & \\ n & 1010 & 343 & 2.91(2.08) & \\ \text { Mean (SD) } & 2.49(2.09) & 1.66(1.88) & & \end{array}$


Table 4 continued

\begin{tabular}{lllll}
\hline Parameter & Overall & Joint-only & Joint and skin & $\boldsymbol{p}$ value* $^{*}$ \\
\hline Median $(\mathrm{IQR})$ & $2.00(0.85,3.55)$ & $1.05(0.20,2.35)$ & $2.60(1.35,3.95)$ & \\
\hline
\end{tabular}

$E Q-5 D$ EuroQol Five Dimension questionnaire; HAQ-DI Health Assessment Questionnaire-Disability Index, IQR interquartile range; PSAID12 Psoriatic Arthritis Impact of Disease questionnaire; $S D$ standard deviation; VAS visual analog scale; WPAI Work Productivity and Activity Impairment

'Student's $t$ test was used to compare outcomes from 'joint-only' with 'joint and skin' groups

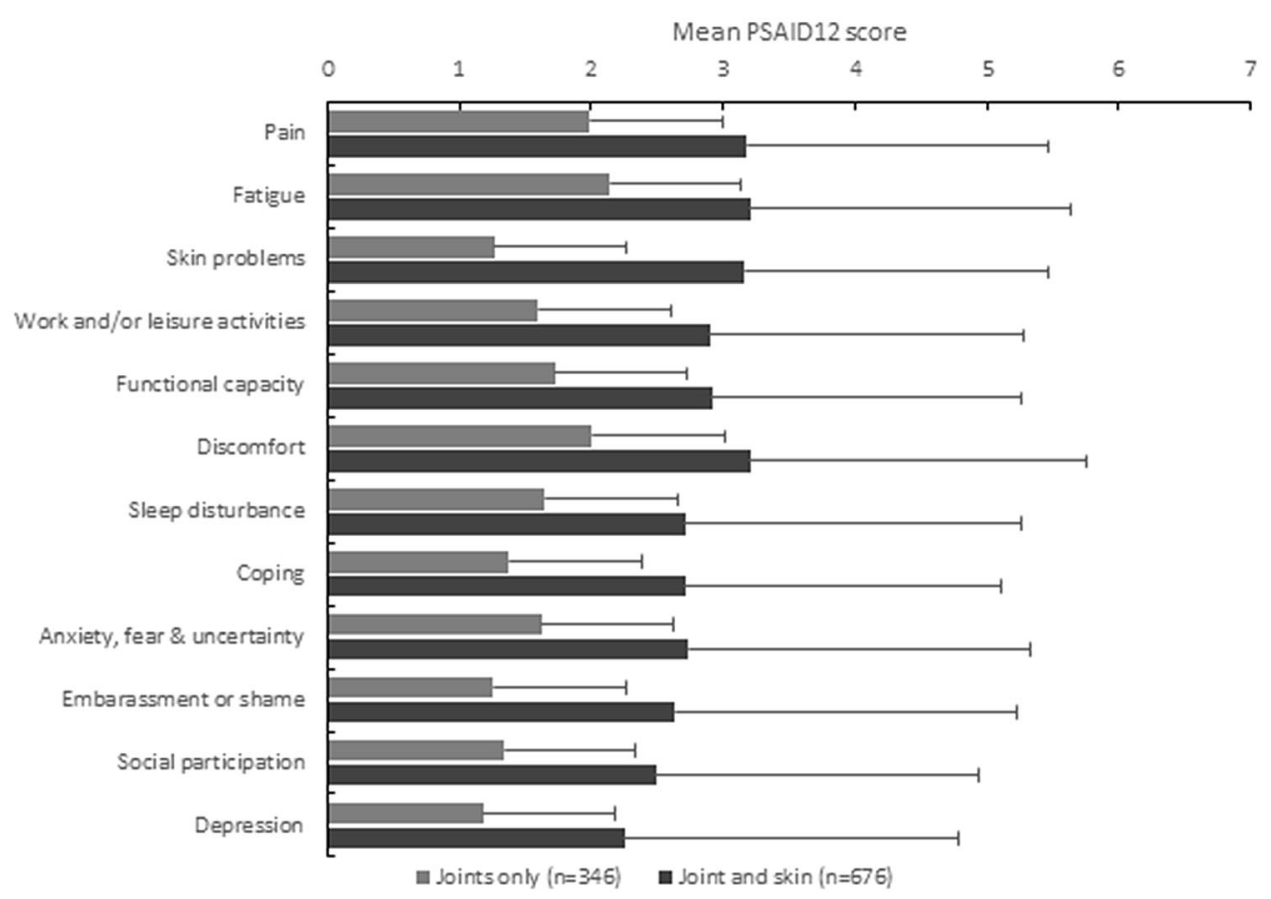

Fig. 2 Analysis of individual PsAID12 scores in patients with 'joint-only' and 'joint and skin' symptoms. Significant $(p<0.001)$ differences between the two groups were seen for all questions making up the PsAID12 questionnaire. The joint only group contained a maximum of 346 patient

\section{Sensitivity Analysis: Clinical \\ Characteristics and Patient-Reported Outcomes in Patients Grouped According to the Number of Affected Joints and for Severity of Skin}

The relative impact on patient-reported outcomes in patients with a physician-assessed joint count of $\leq 4$ vs. $>4$ in patients with 'joint-only' symptoms is shown in Table 5 . Across a range of outcomes patients with four or more affected joints showed significantly worse responses and the joint and skin group contained a maximum of 679 patients. The joint only group contained a minimum of 344 patient responses and the joint and skin group contained a minimum of 672 responses

clinical outcomes ranging from increased disease progression, more current flares, an increase in the mean number of current symptoms, and increased physician-reported pain (Table 5). Alongside clinical outcomes, patientreported outcomes showed a consistent and significant increase in disability, health status, and work impairment with increased number of affected joints.

Outcomes were also assessed in the 1743 patients with joint and skin PsA stratified according to physician-reported skin severity. 
Table 6 shows the clinical characteristics of patients with joint and skin involvement stratified according to physician subjective assessment of skin severity. Patients with joint and skin PsA and severe skin disease showed significantly higher levels of physician-reported joint disease, disease progression and pain levels compared with patients with less severe skin involvement (all $p<0.001)$. Severe joint disease increased from $1.9 \%$ of patients with mild skin disease severity to $21.9 \%$ of patients with severe skin disease while more patients were either unstable $(4.3 \%$ of patients with mild skin disease to $18.5 \%$ and $31.3 \%$ of patients with moderate or severe skin disease) or deteriorating (2.6\%, $9.1 \%$ and $34.3 \%$ of patients with mild, moderate and severe skin disease). Differences in patients managed by rheumatologists and dermatologists are shown in Supplementary Tables 2 and 3.

Current flaring (any flaring of the skin or joint symptoms) in patients with joint and skin involvement was more likely in patients with severe skin symptoms with $45.9 \%$ compared with $4.3 \%$ of patients with mild skin symptoms. Patients with joint and skin symptoms were more likely to have a current flare than jointonly patients but adverse outcomes increased in patients with joint and skin symptoms and with increasing skin disease.

Patient-reported outcomes followed similar trends when patients with joint and skin involvement were stratified according to current skin symptom severity. While base sizes were small in severe categories the general trend was for worse health status (EQ-5D), more disability (HAQ-DI), greater work impairment (WPAI), and a higher impact of disease on health status (PsAID12) with skin severity (all $p<0.001$, except for absenteeism).

\section{DISCUSSION}

In this prospective cross-sectional survey of patients with PsA from nine countries, clinical and patient-reported outcomes were compared in patient groups who had current active skin symptoms (BSA $>0 \%)$ and those who did not have current active skin symptoms $(\mathrm{BSA}=0 \%)$.
All patients were receiving DMARDs and the majority of patients had mild disease severity reported by physicians. Patients who had 'joint and skin' symptoms had more severe forms of the disease, with more symptoms, affected joints, pain, and flaring. The immediate consequences of these clinical characteristics were impaired QoL through greater emotional burden, and worse health status, more disability, greater work impairment, and a higher impact of disease on health status.

The demographic and clinical characteristics of this cohort are comparable with other contemporary real-world studies of PsA patients, including the proportion with $\mathrm{BSA}=0$ and current therapies $[6,13]$. There was a consistent pattern of worse outcomes in patients with active skin symptoms compared with 'jointonly' PsA with higher physician-assessed disease activity including higher joint counts, physician subjective assessment of joint and skin severity, physician-assessed pain, and number of other current symptoms. These results are similar to analyses from a U.S. registry study that found patients with greater skin severity (BSA $>3 \%$ ) were twice as likely to have more severe joint disease, measured by the CDAI score, compared with patients with $\mathrm{BSA}=0 \%$ [6]. Data from a second analysis of the same registry found PsA patients with higher skin severity $(\mathrm{BSA}>3 \%)$ had significantly higher TJC, SJC, DAS28-CRP, and CDAI score compared with patients with BSA $\leq 3 \%$ [13]. This is similar to results from the LOOP study: patients managed by dermatologists were shown to have higher tender and swollen joint counts compared to those seen by rheumatologists [26].

Significant differences in PROs were also seen between 'joint and skin' and 'joint-only' patients, with higher disability (HAQ-DI), worse work productivity (WPAI), and health status (EQ-5D, PsAID) in the 'joint and skin' group. However, patients in the joint-only group were more likely to be receiving a bDMARD, which could contribute to these patients having better outcomes than patients with joint and skin involvement. Assessment of the individual 12 items of the PsAID showed significantly higher (worse) scores across all individual items for the 'joint and skin' compared with the 'joint only' 
Table 5 Clinical and patient-reported outcomes according to number of affected joints in patients with 'joint-only' PsA

\begin{tabular}{lllll}
\hline Parameter & Overall & $\mathbf{5} \mathbf{4}$ affected joints & $>\mathbf{4}$ affected joints & $\boldsymbol{p}$ value \\
\hline$n \%$ & 960 & 853 & 107 &
\end{tabular}

Current overall severity

\begin{tabular}{|c|c|c|c|c|}
\hline Mild & $824(85.8)$ & $770(90.3)$ & $54(50.5)$ & $<0.001^{*}$ \\
\hline Moderate & $124(12.9)$ & $75(8.8)$ & $49(45.8)$ & \\
\hline Severe & $12(1.3)$ & $8(0.9)$ & $4(3.7)$ & \\
\hline
\end{tabular}

Current joint severity

\begin{tabular}{|c|c|c|c|c|}
\hline Mild & $822(85.6)$ & $769(90.2)$ & $53(49.5)$ & $<0.001^{*}$ \\
\hline Moderate & $125(13.0)$ & $77(9.0)$ & $48(44.9)$ & \\
\hline Severe & $13(1.4)$ & $7(0.8)$ & $6(5.6)$ & \\
\hline
\end{tabular}

Disease progression

$\begin{array}{lllll}\text { Improving } & 297(30.9) & 268(31.4) & 29(27.1) & <0.001^{\#} \\ \text { Stable } & 597(62.2) & 547(64.1) & 50(46.7) & \\ \text { Unstable } & 35(3.6) & 19(2.2) & 16(15.0) & 11(10.3) \\ \text { Deteriorating slowly } & 26(2.7) & 15(1.8) & 1(0.9) & <0.001^{\ddagger} \\ \text { Deteriorating rapidly } & 5(0.5) & 4(0.5) & 3.77(1.89) & <0.001^{\ddagger} \\ \begin{array}{l}\text { Pain (1-10) } \\ \text { Mean (SD) }\end{array} & 2.16(1.51) & 1.96(1.32) & & 2.62(1.87) \\ \text { Number of current symptoms } & & & \end{array}$

EQ5D utility score

$\begin{array}{lllll}n & 384 & 333 & 51 & <0.001^{\ddagger} \\ \text { Mean (SD) } & 0.85(0.17) & 0.86(0.17) & 0.77(0.18) & <0.001^{\ddagger} \\ \text { EQ5D VAS score } & & & 52 & \\ n & 389 & 337 & 68.98(20.76) & <0.001^{\ddagger} \\ \text { Mean (SD) } & 77.68(17.51) & 79.02(16.59) & & \\ \text { HAQ-DI } & & 320 & 0.61(0.61) & \end{array}$

WPAI: percent impairment while working due to problem

$\begin{array}{lllll}n & 219 & 194 & 25 & 0.006^{\ddagger} \\ \text { Mean (SD) } & 11.83(15.75) & 10.77(15.06) & 20.00(18.71) & \end{array}$

WPAI: percent overall work impairment due to problem

\begin{tabular}{lllll}
$n$ & 183 & 159 & 24 & $0.006^{*}$ \\
\hline
\end{tabular}


Table 5 continued

\begin{tabular}{lllll}
\hline Parameter & Overall & $\mathbf{5} \mathbf{4}$ affected joints & $\mathbf{>}$ 4 affected joints & $\boldsymbol{p}$ value \\
\hline Mean (SD) & $16.32(20.64)$ & $14.70(19.57)$ & $27.07(24.52)$ & \\
WPAI: percent activity & impairment due to problem & & & \\
$n$ & 385 & 333 & 52 & $0.001^{\ddagger}$ \\
Mean (SD) & $19.48(22.33)$ & $18.02(21.85)$ & $28.85(23.32)$ & \\
PsAID12 score (0-10) & & & 45 & $0.001^{\ddagger}$ \\
$n$ & 343 & 298 & $2.68(1.96)$ & \\
Mean (SD) & $1.66(1.86)$ & $1.50(1.80)$ & & \\
\hline
\end{tabular}

EQ-5D EuroQol Five Dimension questionnaire; HAQ-DI Health Assessment Questionnaire-Disability Index, PsAID12 Psoriatic Arthritis Impact of Disease questionnaire; $S D$ standard deviation; VAS visual analog scale; WPAI Work Productivity and Activity Impairment

*Mann Whitney test. ${ }^{\#}$ Chi squared test; ${ }^{\star}$ Student's $t$ test

groups. Fatigue, discomfort, pain, and skin problems had the highest impact in the joint and skin group, while pain, fatigue, discomfort, and functional capacity were most impactful in the 'joint only' group. Similar results on PROs were seen in the previously mentioned U.S. registry study where PsA patients with BSA > $3 \%$ had higher HAQ-DI, higher pain and fatigue scores, and worse work productivity (WPAI) compared with patients with BSA $\leq 3 \%$ [13].

When asked to prioritize which symptom (joint or skin) patients would rather be rid of, given the choice of only one, the majority of patients (> 60\%) chose joint symptoms. While approximately $40 \%$ of patients in the 'joint and skin' group selected skin, it is interesting that $35 \%$ in the 'joint only' group also prioritized skin symptoms. This response may indicate that each group of patients differs in disease severity rather than representing different domains of the disease, and this is reflected in PROs and clinical outcomes. Other studies have shown that skin symptoms can have a high priority to a subset of PsA patients. In the Disconnect study, an online survey was used to assess the perceived bother of joint and skin symptoms to PsA patients $(n=200)$. Using a best-worst scaling methodology, the investigators found the most bothersome symptom identified by the patients who completed the survey was painful, inflamed, or broken skin, followed by joint pain, soreness, or tenderness [27]. Patient surveys done during the development of the PsAID instrument also found that skin symptoms, in addition to pain and fatigue were the three most impactful symptoms identified by patients [24].

Prioritization of one symptom over another could at least in part be related to the emotional burden while it is likely that collective contribution of all symptoms will contribute to the burden of disease. Previous studies have identified the psoriasis component of PsA with an extra, negative, impact on HR-QoL due to its effects on psychosocial health. In this study, approximately $40 \%$ of patients indicated they experience anxiety or depression related to their PsA, with no statistically significant differences between 'joint and skin' compared with 'joint only' group. The majority of patients (62\%) indicated both joint and skin symptoms contributed to the anxiety/depression.

Worse outcomes were seen in patients with 'joint and skin' involvement compared with 'joint-only' diagnoses while increased skin severity within the 'joint and skin' cohort of patients was accompanied by a further decline in health status. Additional analysis showed that patients with 'joint and skin' disease are more impacted than those with 'joint-only' disease with many PROs showing a dependence on the number of affected joints. Not unexpectedly there was a decline in health status/ 
Table 6 Clinical characteristics and patient reported outcomes in patients with joint and skin PsA stratified according to physician-reported skin severity

\begin{tabular}{llllll}
\hline Parameter & Overall & Mild & Moderate & Severe & $\boldsymbol{p}$ value \\
\hline$n \%$ & $1743(100.0)$ & $1240(71.1)$ & $439(25.2)$ & $64(3.7)$ &
\end{tabular}

Overall severity, $n(\%)$

Mild

Moderate

Severe

Joints severity, $n(\%)$

Mild

Moderate

Severe

Disease status, $n(\%)$

Improving

Stable

Unstable

Deteriorating slowly

Deteriorating rapidly

Does patient experience flares? $n$ (\%)

n 1710

Yes, currently experiencing a flare

Yes, within the last 12 months but not currently

Yes, but not within the last

12 months

Pain (1-10)

Mean (SD)

Median (IQR)

EQ-5D utility score

n

Mean (SD)

Median (IQR)

EQ-5D VAS score

$\begin{array}{llll}1207(69.2) & 1068(86.1) & 124(28.2) & 15(23.4) \\ 473(27.1) & 154(12.4) & 295(67.2) & 24(37.5) \\ 63(3.6) & 18(1.5) & 20(4.6) & 25(39.1)\end{array}$

$\begin{array}{llll}1222(70.1) & 1045(84.3) & 161(36.7) & 16(25.0)\end{array} \quad<0.001^{*}$

$\begin{array}{llll}627(36.0) & 480(38.7) & 142(32.3) & 5(7.8)\end{array} \quad<0.001^{\#}$

$150(8.8)$

$446(26.1)$

$469(27.4)$

$372(30.5)$

$3.08(1.79)$

$3.00(2.00$,

4.00)

830

$0.79(0.17)$

$0.81(0.72$,

$0.91)$

839

590

$52(4.3)$

$301(24.7)$

584

0.83 (0.74,

$0.92)$
428

70 (16.4)

61

$<0.001^{\#}$

$135(31.5)$

28 (45.9)

$10(16.4)$

$91(21.3)$

$6(9.8)$

$$
\begin{aligned}
& 2.63(1.49) \\
& 2.00(2.00 \\
& 3.00)
\end{aligned}
$$

4.01 (1.85)

$5.47(2.28)$

$<0.001^{*}$

4.00 (3.00,

5.00)

$6.00(4.00,7.00)$

$0.81(0.16)$

219

$0.74(0.18)$

27

$<0.001$

$0.76(0.68$,

$0.83)$
$0.71(0.25)$

$0.81(0.63,0.89)$

n

(2)

221

28

$<0.001^{*}$ 
Table 6 continued

\begin{tabular}{llllll}
\hline Parameter & Overall & Mild & Moderate & Severe & $\boldsymbol{p}$ value \\
\hline Mean (SD) & $71.98(19.01)$ & $74.29(19.03)$ & $67.12(17.47)$ & $61.71(20.23)$ & \\
Median (IQR) & $75.00(65.00$, & $80.00(65.00$, & $70.00(60.00$, & $66.50(50.00$, & \\
& $85.00)$ & $90.00)$ & $80.00)$ & $77.50)$ &
\end{tabular}

HAQ Disability Index

$\begin{array}{llllll}n & 805 & 566 & 214 & 25 & <0.001^{*} \\ \text { Mean (SD) } & 0.57(0.59) & 0.50(0.56) & 0.76(0.63) & 0.67(0.64) & \\ \text { Median (IQR) } & 0.38(0.00, & 0.25(0.00, & 0.75(0.13, & 0.38(0.13,1.13) & \end{array}$

WPAI: percent work time missed due to problem

$\begin{array}{llllll}n & 381 & 260 & 108 & 13 & 0.075^{\ddagger} \\ \text { Mean (SD) } & 4.74(16.20) & 3.52(14.28) & 7.73(20.43) & 4.34(8.40) & \\ \text { Median (IQR) } & 0.0(0.00,0.00) & 0.0(0.00,0.00) & 0.0(0.00,0.00) & 0.0(0.00,0.00)\end{array}$

WPAI: Percent impairment while working due to problem

$\begin{array}{llllll}n & 411 & 286 & 112 & 13 & <0.001^{*} \\ \text { Mean (SD) } & 23.26(18.87) & 19.65(16.92) & 30.27(19.33) & 42.31(27.43) & \\ \text { Median (IQR) } & 20.00(10.00, & 20.00(10.00, & 30.00(20.00, & 40.00(20.00, & \end{array}$

WPAI: percent overall work impairment due to problem

$\begin{array}{llllll}n & 373 & 256 & 104 & 13 & <0.001^{*} \\ \text { Mean (SD) } & 25.61(20.51) & 21.36(18.17) & 33.83(21.31) & 43.60(28.39) & \\ \text { Median (IQR) } & 20.00(10.00, & 20.00(10.00, & 30.00(20.00, & 40.00 \\ & 30.00) & 30.00) & 50.00) & (20.00-60.53)\end{array}$

WPAI: percent activity impairment due to problem

$\begin{array}{llllll}n & 819 & 578 & 217 & 24 & <0.001^{*} \\ \text { Mean (SD) } & 29.99(22.81) & 26.45(21.79) & 37.33(22.45) & 48.75(25.93) & \\ \text { Median (IQR) } & 30.00(10.00, & 20.00(10.00, & 30.00(20.00, & 50.00(30.00, & \\ & 40.00) & 40.00) & 50.00) & 60.00) & <0.001^{*} \\ \text { PsAID12 score (0-10) } & & & & 26 & \\ n & 667 & 455 & 186 & 4.84(2.25) & \\ \text { Mean (SD) } & 2.91(2.08) & 2.47(1.93) & 3.71(2.03) & & \\ \text { Median (IQR) } & 2.60(1.35, & 2.00(1.05, & 3.40(2.30, & 4.68(3.65,6.35) & \end{array}$

$I Q R$ interquartile range; $S D$ standard deviation

* Kruskal-Wallace test; ${ }^{\#}$ Chi-squared test; ${ }^{*}$ ANOVA were used to compare outcomes across severity groups 
QoL/work activity with increasing number of affected joints in the 'joint-only' patient population. In 'joint and skin' patients, worse skin symptoms had a greater adverse impact on QoLrelated health status, disability, and WPAI-related outcomes compared with those with milder or less severe skin symptoms.

Severe skin symptoms and additional number of affected joints clearly have a detrimental impact on outcomes in the respective patient populations. There is some evidence to suggest that greater skin severity in the 'joint and skin' group was associated with worse outcomes compared with greater number of joints affected in the 'joint-only' group although this was not a formalized comparison. There was a consistent trend for patients with greater skin severity to show worse outcomes than patients with $>4$ affected joints. For example, the overall disease severity in the 'joint-only' group with $>4$ affected joints showed that $3.7 \%$ of patients were assessed as severe compared with $39.1 \%$ of patients in the severe skin group. Joint severity in the 'joint-only' group with $>4$ joints showed that physicians evaluated $5.6 \%$ as severe compared with $21.9 \%$ in the severe skin group. Physician-reported pain scores were higher at 5.47 in patients in the severe skin groups compared with 3.77 in the 'joint-only' cohort with $>4$ affected joints. Patient-reported outcomes also showed this trend with an HAQ-DI score of 0.61 in 'joint-only' patients with $>4$ affected joints compared with 0.67 in the severe skin group, an EQ-5D utility score of 0.77 in patients with $>4$ affected joints compared with 0.71 in patients with severe skin symptoms and a PsAID score in these two groups of 2.68 and 4.84 , respectively.

The results obtained here in real-world patient populations are complemented by studies of clinical trial populations that showed that optimal improvements in patients' HR-QoL were dependent on successful treatment of both joint and skin symptoms $[28,29]$.

\section{LIMITATIONS}

These limitations are related to the non-randomized selection of patients by physicians who were asked to choose a consecutive series of patients to avoid selection bias. Selection bias may still exist due to, for example, the physicians who chose to participate in the survey, location of practice, or the disease severity. No formal source data verification procedures were implemented. The patients were only representative of those currently actively seeking treatment so there is a potential over-representation of well-motivated patients or of patients with less severe PsA. Inclusion and exclusion criteria were not extensive, diagnosis of the target patient group is based on judgement of the respondent physician and not a formalized and validated diagnostic checklist. In addition, this study focused on a limited number of outcomes as disease-specific scores were not described in this study and there was no analysis of enthesitis, dactylitis, axial involvement, or nail involvement in this study although these outcomes were collected for future analysis. The cross-sectional design cannot be used to demonstrate cause and effect and all data were purely descriptive with no covariate adjustment. However, these limitations should be balanced with the methodological strengths which include recruitment of a large, representative sample of patients with PsA across geographies, capturing patients from specialty physician practices, and standardized data collection tools across countries.

\section{CONCLUSIONS}

In this real-world, non-interventional, crosssectional survey of patients with PsA, those with 'joint and skin' involvement experienced worse health outcomes including more flaring, increased emotional burden, and decreased health-related QoL compared with patients with 'joint-only' PsA. Not only was there a significant impact on QoL but work productivity declined, and disability increased suggesting that future treatment options in patients with PsA must focus on both skin and joint symptoms to optimize patient outcomes and minimize QoL/productivity impact. Health status, productivity, and disability were further adversely affected by increasing skin severity in 
patients with 'joint and skin' involvement, suggesting that additional effort and enhanced future treatment approaches should also focus on ameliorating disease progression in this group of patients in routine real-world practice.

\section{ACKNOWLEDGEMENTS}

Funding. This study and the Rapid Service Fee were funded by Eli Lilly and Company (Indianapolis, IN, USA). All authors had full access to all of the data in this study and take complete responsibility for the integrity of the data and accuracy of the data analysis.

Editorial Assistance. Thanks to David Whitford of Sapitwa Communications for medical writing support. Adelphi was paid a fee by Eli Lilly for the manuscript production (joint authorship venture), and Adelphi used this funding to obtain the medical writing support for this article.

Authorship. All named authors meet the International Committee of Medical Journal Editors (ICMJE) criteria for authorship for this article, take responsibility for the integrity of the work as a whole, and have given their approval for this version to be published.

Disclosures. William Tillett: Grant/research support from: AbbVie, Celgene, and Lilly, Consultant for: AbbVie, Amgen, Celgene, Lilly, Novartis, Pfizer, and UCB Speakers bureau: AbbVie, Celgene, Lilly, Janssen, Novartis, UCB, and Pfizer. Joseph F. Merola is a consultant and/ or investigator for Merck, AbbVie, Dermavant, Eli Lilly, Novartis, Janssen, UCB, Celgene, Sanofi, Regeneron, Arena, Sun Pharma, Biogen, Pfizer, EMD Serono, Avotres and Leo Pharma. Diamant Thaçi has been a consultant and advisor and/or received speaking fees and/or grants and/or served as an investigator in clinical trials for the following companies: AbbVie, Almirall, Amgen, Asana, Bioskin, Biogen, Boehringer Ingelheim, Bristol-Myers-Squib, Celgene, Dermira, DS-Biopharma, Eli Lilly, Galapagos, Galderma, LEO Pharma, Janssen-
Cilag, Kymab, Merck Sharp Dohme, Morphosis, Novartis, Pfizer, Regeneron, Roche, Sandoz, Sanofi, Samsung and UCB. Wolf-Henning Boehncke has received honoraria as a speaker and/or advisor from the following companies: AbbVie, Amgen, Almirall, Celgene, Janssen, Leo, Lilly, Novartis, UCB. Matthew Hufford and Julie Birt are employees and shareholders of Eli Lilly. Nicola Booth, Elizabeth Holdsworth, Steve Lobosco and Gary Milligan are employees of Adelphi Real World.

Compliance with Ethics Guidelines. The non-interventional, observational nature of the data collection does not result in patients being placed at risk from the study. Patients provided informed consent to participate in the study and did not provide any personally identifiable information. All responses were anonymized to preserve respondent (physician and patient) confidentiality and all participating physicians and patients were assigned a study number to aid anonymous data collection and to allow linkage of data during data collection and analysis. The research was conducted in accordance with national market research and privacy regulations (EphMRA, U.S. Department of Health and Human Services National Institutes of Health, HIPAA). Ethical approvals were sought and granted through Western-IRB (Study ID number 1183030) for the US \& Canada, and through Freiburg Ethics Commission (FEKI) for all other countries.

Data Availability. All data analyzed during this study are included in the published article or as supplementary information files or can be made available on request.

Open Access. This article is licensed under a Creative Commons Attribution-NonCommercial 4.0 International License, which permits any non-commercial use, sharing, adaptation, distribution and reproduction in any medium or format, as long as you give appropriate credit to the original author(s) and the source, provide a link to the Creative Commons licence, and indicate if changes were made. The images or other third party material in this article are included in the article's 
Creative Commons licence, unless indicated otherwise in a credit line to the material. If material is not included in the article's Creative Commons licence and your intended use is not permitted by statutory regulation or exceeds the permitted use, you will need to obtain permission directly from the copyright holder. To view a copy of this licence, visit http:// creativecommons.org/licenses/by-nc/4.0/.

\section{REFERENCES}

1. Christophers E, Barker JN, Griffiths CE, et al. The risk of psoriatic arthritis remains constant following initial diagnosis of psoriasis among patients seen in European dermatology clinics. J Eur Acad Dermatol Venereol. 2010;24(5):548-54.

2. Ritchlin CT, Colbert RA, Gladman DD. Psoriatic arthritis. N Engl J Med. 2017;376(21):2095-6.

3. Coates LC, Helliwell PS. Psoriatic arthritis: state of the art review. Clin Med (Lond). 2017;17(1):65-70.

4. Gladman DD, Farewell V, Buskila D, et al. Reliability of measurements of active and damaged joints in psoriatic arthritis. J Rheumatol. 1990;17(1):62-4.

5. McHugh NJ, Balachrishnan C, Jones SM. Progression of peripheral joint disease in psoriatic arthritis: a 5-yr prospective study. Rheumatology (Oxford). 2003;42(6):778-83.

6. Mease PJ, Etzel CJ, Huster WJ, et al. Understanding the association between skin involvement and joint activity in patients with psoriatic arthritis: experience from the Corrona Registry. RMD Open. 2019;5(1):e000867.

7. Merola JF, Shrom D, Eaton J, et al. Patient perspective on the burden of skin and joint symptoms of psoriatic arthritis: results of a multi-national patient survey. Rheumatol Ther. 2019;6(1):33-45.

8. Rosen CF, Mussani F, Chandran V, et al. Patients with psoriatic arthritis have worse quality of life than those with psoriasis alone. Rheumatology (Oxford). 2012;51(3):571-6.

9. Salaffi F, Carotti M, Gasparini S, et al. The healthrelated quality of life in rheumatoid arthritis, ankylosing spondylitis, and psoriatic arthritis: a comparison with a selected sample of healthy people. Health Qual Life Outcomes. 2009;7:25.
10. Zachariae $H$, Zachariae R, Blomqvist $\mathrm{K}$, et al. Quality of life and prevalence of arthritis reported by 5,795 members of the Nordic Psoriasis Associations. Data from the Nordic Quality of Life Study. Acta Derm Venereol. 2002;82(2):108-13.

11. Husted JA, Gladman DD, Farewell VT, Cook RJ. Health-related quality of life of patients with psoriatic arthritis: a comparison with patients with rheumatoid arthritis. Arthritis Rheum. 2001;45(2): 151-8.

12. de Vlam K, Merola JF, Birt JA, et al. Skin involvement in psoriatic arthritis worsens Dooverall disease activity, patient-reported outcomes, and increases healthcare resource utilization: an observational. Cross-Sect Study Rheumatol Ther. 2018;5(2):423-36.

13. Mease PJ, Karki C, Palmer JB, et al. Clinical and patient-reported outcomes in patients with Psoriatic Arthritis (PsA) by body surface area affected by psoriasis: results from the corrona PsA/Spondyloarthritis Registry. J Rheumatol. 2017;44(8):1151-8.

14. Kamalaraj N, El-Haddad C, Hay P, Pile K. Systematic review of depression and anxiety in psoriatic arthritis. Int J Rheum Dis. 2019;22(6):967-73.

15. Moverley AR, Vinall-Collier KA, Helliwell PS. It's not just the joints, it's the whole thing: qualitative analysis of patients' experience of flare in psoriatic arthritis. Rheumatology (Oxford). 2015;54(8): 1448-533.

16. Moverley AR, Waxman R, de Wit M, et al. Development of a flare instrument for use in psoriatic disease: a report from the 2015 GRAPPA annual meeting. J Rheumatol. 2016;43(5):974-8.

17. Anderson P, Benford M, Harris N, et al. Real-world physician and patient behaviour across countries: disease-specific programmes-a means to understand. Curr Med Res Opin. 2008;24(11):3063-72.

18. Dolan P. Modeling valuations for EuroQol health states. Med Care. 1997;35(11):1095-108.

19. EuroQol G. EuroQol-a new facility for the measurement of health-related quality of life. Health Policy. 1990;16(3):199-208.

20. Reilly MC, Zbrozek AS, Dukes EM. The validity and reproducibility of a work productivity and activity impairment instrument. Pharmacoeconomics. 1993;4(5):353-65.

21. Tillett W, Lin CY, Zbrozek A, et al. A threshold of meaning for work disability improvement in psoriatic arthritis measured by the work productivity and activity impairment questionnaire. Rheumatol 
Ther. 2019. https://doi.org/10.1007/s40744-0190155-5.

22. Fries JF, Spitz P, Kraines RG, Holman HR. Measurement of patient outcome in arthritis. Arthritis Rheum. 1980;23(2):137-45.

23. Fries JF, Spitz PW, Young DY. The dimensions of health outcomes: the health assessment questionnaire, disability and pain scales. J Rheumatol. 1982;9(5):789-93.

24. Gossec L, de Wit M, Kiltz U, et al. A patient-derived and patient-reported outcome measure for assessing psoriatic arthritis: elaboration and preliminary validation of the Psoriatic Arthritis Impact of Disease (PsAID) questionnaire, a 13-country EULAR initiative. Ann Rheum Dis. 2014;73(6):1012-9.

25. Orbai AM, Ogdie A. Patient-reported outcomes in psoriatic arthritis. Rheum Dis Clin North Am. 2016;42(2):265-83.

26. Boehncke WH, Horvath R, Dalkilic E, et al. Association between clinical specialty setting and disease management in patients with psoriatic arthritis: results from LOOP, a cross-sectional, multi-country, observational study. J Eur Acad Dermatolo Venereol. 2020. https://doi.org/10.1111/jdv.16251.

27. Husni ME, Fernandez A, Hauber B, et al. Comparison of US patient, rheumatologist, and dermatologist perceptions of psoriatic disease symptoms: results from the DISCONNECT study. Arthritis Res Ther. 2018;20(1):102.

28. Kavanaugh A, Gottlieb A, Morita A, et al. The contribution of joint and skin improvements to the health-related quality of life of patients with psoriatic arthritis: a post hoc analysis of two randomised controlled studies. Ann Rheum Dis. 2019;78(9): 1215-9.

29. Kavanaugh A, McInnes IB, Krueger GG, et al. Patient-reported outcomes and the association with clinical response in patients with active psoriatic arthritis treated with golimumab: findings through 2 years of a phase III, multicenter, randomized, double-blind, placebo-controlled trial. Arthritis Care Res (Hoboken). 2013;65(10):1666-733. 